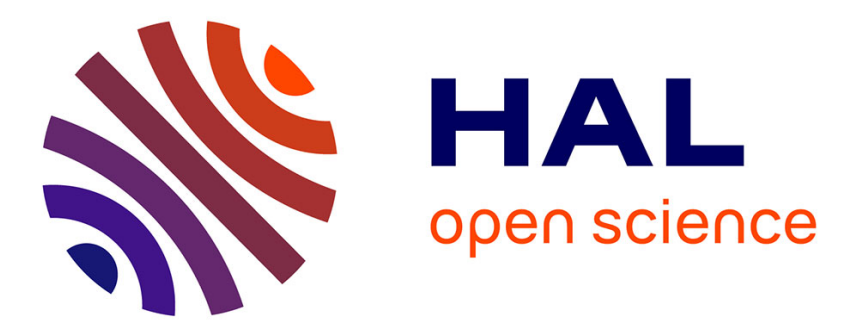

\title{
Generalization of a Correlation Method for Time-Delay Estimation with Application to a River Reach
}

Magalie Thomassin, Thierry Bastogne, Alain Richard, Hugues Garnier

\section{To cite this version:}

Magalie Thomassin, Thierry Bastogne, Alain Richard, Hugues Garnier. Generalization of a Correlation Method for Time-Delay Estimation with Application to a River Reach. 14th IFAC Symposium on System Identification, SYSID 2006, March, Mar 2006, Newcastle, Australia. pp.CDROM. hal00086716

\section{HAL Id: hal-00086716 https://hal.science/hal-00086716}

Submitted on 19 Jul 2006

HAL is a multi-disciplinary open access archive for the deposit and dissemination of scientific research documents, whether they are published or not. The documents may come from teaching and research institutions in France or abroad, or from public or private research centers.
L'archive ouverte pluridisciplinaire HAL, est destinée au dépôt et à la diffusion de documents scientifiques de niveau recherche, publiés ou non, émanant des établissements d'enseignement et de recherche français ou étrangers, des laboratoires publics ou privés. 


\title{
GENERALIZATION OF A CORRELATION METHOD FOR TIME-DELAY ESTIMATION WITH APPLICATION TO A RIVER REACH
}

\author{
Magalie Thomassin* Thierry Bastogne* Alain Richard* \\ * Centre de Recherche en Automatique de Nancy (CRAN) \\ UMR 7039 CNRS-UHP-INPL \\ Université Henri Poincaré, Nancy 1, BP 239 \\ 54506 Vandouvre-lès-Nancy Cedex, France \\ forename.name@cran.uhp-nancy. fr \\ http://www.cran.uhp-nancy.fr
}

\begin{abstract}
This article addresses the problem of time-delay estimation in the specific case of structures with combined feedback/feedforward control. In the case of closed loop single-input/single-output systems, several methods have been developed in order to estimate the time-delay. In particular, this parameter may be determined thanks to a correlation analysis proposed by Zheng and Feng (1990). The purpose of this work is to generalize the latter approach to the case of feedback/feedforward systems (with two inputs: the control input and the measured disturbance). This is motivated by an application to a managed river reach in passive experimental conditions.
\end{abstract}

Copyright (c) 2005 IFAC

Keywords: time-delay estimation, correlation functions, feedback loops, feedforward compensation, hydroelectric systems.

\section{INTRODUCTION}

The time-delay of a system is a crucial parameter to know for many applications. So the time-delay estimation has aroused great interest. One can refer, for instance, to survey papers such as De Souza et al. (1988), Ferreira and Fernandes (1997) and Björklund (2003). In addition, an overview of some recent advances and open problems about time-delay systems may be found in (Richard, 2003). However, little attention has been paid so far to the need for estimation of time-delay of systems with complex structure especially when the system operate under combined feedback/feedforward control. Indeed, in this case, correlations between disturbances and inputs must be taken into account (Söderström, 1999).

Zheng and Feng (1990) developed a time-delay estimation method based on correlation analysis allowing one to consider a closed-loop structure. So the idea has been to generalize this method to structures with combined feedback/feedforward control. This development is motivated by an application: the estimation of a time-delay of a managed river reach in passive experimental conditions (Thomassin et al., 2003; Thomassin et al., 2004; Thomassin, 2005).

This paper is organized as follows. Section 2 reminds the time-delay estimation method developed by Zheng and Feng (1990). Then, in the following section, the river reach modelling is presented to show the particular structure of the application and to highlight difficulties in the identification of the process. Section 4 is devoted to the major contribution of this work, i.e it contains the generalization of the time-delay estimation method (presented in section 2) to structures with combined feedback/feedforward control. Finally, simulation results are presented in section 5 in order to show the effectiveness of the method. 


\section{TIME-DELAY ESTIMATION BY CORRELATION ANALYSIS}

The time-delay estimation from correlation analysis was presented for the first time by Faure and Evans (1969) in a deterministic case. Then, many time-delay estimation methods using correlation functions was developed (Carter, 1987). The first paper presenting a method based on correlation functions allowing the identification of a stochastic system with unknown delay, and correlation between disturbances and input, was Zheng and Feng (1990).

The authors consider the following model:

$$
A\left(q^{-1}\right) y[k]=q^{-d} B\left(q^{-1}\right) u[k]+\omega[k],
$$

where

$$
\begin{aligned}
& A\left(q^{-1}\right)=1-a_{1} q^{-1}-\ldots-a_{n_{a}} q^{-n_{a}}, \\
& B\left(q^{-1}\right)=b_{0}+b_{1} q^{-1}+\ldots+b_{n_{b}} q^{-n_{b}},
\end{aligned}
$$

with $b_{0} \neq 0$ and where $q^{-1}$ is the shift operator. The orders $n_{a}$ and $n_{b}$ are known. The sequences $\{u[k]\}_{k=0}^{N-1}$ and $\{y[k]\}_{k=0}^{N-1}$ denote the system input and observed noisy output. The sequence $\{\omega[k]\}_{k=0}^{N-1}$ represents noises and disturbances acting on the system ( $N$ is the data length). The parameter $d>0$ is the unknown time-delay. It is assumed that the input has a sufficient persistent excitation order so as to respect identifiability conditions. All the zeros of $A\left(q^{-1}\right)$ are outside the closed unit disc (the system is stable).

First, the authors show that if the sequences $\{u[k]\}_{k=0}^{N-1}$ and $\{\omega[k]\}_{k=0}^{N-1}$ are not correlated, and $\{u[k]\}_{k=0}^{N-1}$ is a Moving Average (MA) process of order $n_{f}$ :

$$
u[k]=F\left(q^{-1}\right) r[k],
$$

where:

- $\{r[k]\}_{k=0}^{N-1}$ is a independent identically-distributed (i.i.d.) random sequence; their probability distribution is a zero-mean Gaussian distribution with variance $\sigma^{2}$;

- $F\left(q^{-1}\right)$ denotes the following polynomial:

$$
F\left(q^{-1}\right)=f_{0}+f_{1} q^{-1}+\ldots+f_{n_{f}} q^{-n_{f}},
$$

with $f_{0} \neq 0$ and $f_{n_{f}} \neq 0$,

then the cross-correlation between the input and the output satisfies the following equations:

$$
\left\{\begin{array}{l}
c_{y u}[l]=0, \text { for } l<d-n_{f} \\
c_{y u}\left[d-n_{f}\right]=b_{0} f_{0} f_{n_{f}} \sigma^{2} \neq 0 .
\end{array}\right.
$$

Consequently, the time-delay $d$ can be estimated from the estimates of the cross-correlation function between the output and the input, $\hat{c}_{y u}$, and the autocorrelation order of the input, $\hat{n}_{f}$. This estimation is obtained by the determination of the lag from which the crosscorrelation function is higher than a threshold. It is also possible to use an algorithm which detects a change in the mean such as the CUSUM (cumulative sum) algorithm (Basseville and Nikiforov, 1993).
Once the time-delay is estimated, the model parameters can be estimated by using the least square method with the shifted input $\{u[k-\hat{d}]\}_{k=0}^{N-1}$.

Nevertheless, the major contribution presented in (Zheng and Feng, 1990) is the extension of the previous propriety to the case of correlation between the input and the process noise, i.e. the extension to the closed-loop case. In order to consider this problem, they assume that the cross-correlation between $\{u[k]\}_{k=0}^{N-1}$ and $\{\omega[k]\}_{k=0}^{N-1}$ verifies the following expression $^{1}$ :

$$
\left\{\begin{array}{l}
c_{\omega u}[l] \neq 0, \quad-m \leqslant l \leqslant 0, \\
c_{\omega u}[l]=0, \quad l<-m \text { or } l>0,
\end{array}\right.
$$

where $m \geqslant 0$ is the cross-correlation order between $\omega$ and $u$. It means that the control at time $k$ depends only on the disturbances at times $k, k-1, \ldots, k-$ $m$. Then, if $\{u[k]\}_{k=0}^{N-1}$ is a stationary uncorrelated random sequence with zero mean and variance $\sigma_{u}^{2}$ and $\{\omega[k]\}_{k=0}^{N-1}$ is a white noise, they show that the coefficients $a_{i}$ can be estimated thanks to the following equations:

$$
c_{y y}[l]=\sum_{i=1}^{n_{a}} a_{i} c_{y y}[l-i],
$$

for $l>n_{b}+d+m$, where $m$ can be estimated from equation (4) and $d$ can be bounded by a maximal value $d_{\text {max }}$. These equations are named generalised YuleWalker equations. The demonstration can be found in the article (Zheng and Feng, 1990).

Once the coefficients $a_{i}$ are estimated, it is possible to calculate the following expression:

$$
\hat{\rho}_{y u}[l] \triangleq c_{y u}[l]-\sum_{i=1}^{n_{a}} \hat{a}_{i} c_{y u}[l-i],
$$

which is theoretically equal to:

$$
\rho_{y u}[l]=\sum_{i=0}^{n_{b}} b_{i} c_{u u}[l-i]+c_{\omega u}[l],
$$

and, consequently, satisfies the following conditions:

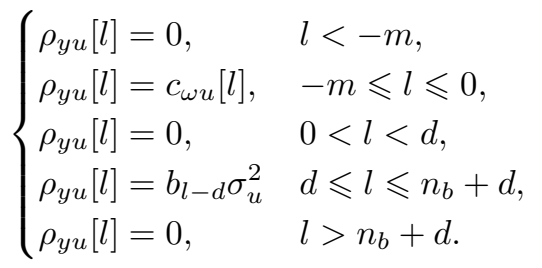

The time-delay $d$ can also be obtained by detecting the first positive lag which verifies $\hat{\rho}_{y u}[l] \neq 0$.

Let us reconsider the assumption that the input $\{u[k]\}_{k=0}^{N-1}$ is a uncorrelated random sequence. If we assume that $\{\omega[k]\}_{k=0}^{N-1}$ is a white noise and that the cross-correlation function between $\omega$ and $u$ verifies

\footnotetext{
1 This assumption is rather restrictive. Indeed, it is not checked if the input is not a white noise. In this case, there will be as well as possible $c_{\omega u}[l] \neq 0$ for $-m \leqslant l \leqslant n_{\omega}$, where $n_{\omega}>0$ is the autocorrelation order of $\omega$. Nevertheless, the extension to this assumption is easy to developed.
} 
equation (4), i.e. that $u[k]$ depends on $\{\omega[k], \omega[k-$ $1], \ldots, \omega[k-m]\}$, then $u[k]$ depends on $\{u[k], u[k-$ $1], \ldots, u[k-m]\}$ and the assumption about the noncorrelation of $\{u[k]\}_{k=0}^{N-1}$ is false. Moreover, in the case of closed-loop structure, this assumption is not realistic: the control at time $k$ depends at least on that at time $k-1$. At best, one can assume that the autocorrelation function of $u$ is finite, of order $n_{f}>0$ :

$$
\begin{cases}c_{u u}[l] \neq 0, & |l| \leqslant n_{f}, \\ c_{u u}[l]=0, & l<-n_{f} \text { or } l>n_{f} .\end{cases}
$$

Then, by a similar demonstration, by always supposing that $\{\omega[k]\}_{k=0}^{N-1}$ is a white noise and that the crosscorrelation function between $\omega$ and $u$ verifies the relation (4), we show the following property:

$$
c_{y y}[l]=\sum_{i=1}^{n_{a}} a_{i} c_{y y}[l-i],
$$

for $l>n_{b}+d+\max \{m, p-d\}$. These equations make it possible to estimate the coefficients $a_{i}$, which allow one to estimate the statistic $\hat{\rho}_{y u}[l]$ always defined by equation (6), which now verifies the conditions:

$$
\begin{cases}\rho_{y u}[l]=0, & l<-m, \\ \rho_{y u}[l] \neq 0, & -m \leqslant l \leqslant 0, \\ \rho_{y u}[l]=0, & 0<l<d-n_{f}, \\ \rho_{y u}[l] \neq 0 & d-n_{f} \leqslant l \leqslant n_{b}+d+n_{f}, \\ \rho_{y u}[l]=0, & l>n_{b}+d+n_{f} .\end{cases}
$$

This statistic allows to estimate the lag $d-n_{f}$ (under the condition $d-n_{f}>0$ ) or $n_{b}+d+n_{f}$. So the delay $d$ can be estimated (if $n_{f}$ is not a priori known, it is possible to estimate it from the autocorrelation function of $u$ ).

Remark 1 If $\{\omega[k]\}_{k=0}^{N-1}$ is not a white noise, it is also possible to develop a similar theory by assuming that :

$$
\begin{cases}c_{\omega \omega}[l] \neq 0, & |l| \leqslant n_{\omega}, \\ c_{\omega \omega}[l]=0, & l<-n_{\omega} \text { or } l>n_{\omega},\end{cases}
$$

i.e. $\{\omega[k]\}_{k=0}^{N-1}$ is a MA process of order $n_{\omega}$ :

$$
\omega[k]=\Omega\left(q^{-1}\right) e[k],
$$

with $\Omega\left(q^{-1}\right)=\omega_{0}+\omega_{1} q^{-1}+\cdots+\omega_{n_{\omega}} q^{-n_{\omega}}$, and where $\{e[k]\}_{k=0}^{N-1}$ is a white noise. $\diamond$

In conclusion, it is possible to estimate the timedelay of a single-input/single-output structure from this method even if the input/output data are correlated. In section 4, we will show that it is possible to generalize the principle of this method to the case of a structure having two inputs and an output correlated by a combined feedback/feedforward control.

\section{THE RIVER REACH PROCESS}

The application which led us to the generalization of the latter method is a managed river reach producing hydroelectric power. As depicted in figure 1, the process is composed of a river portion with upstream and

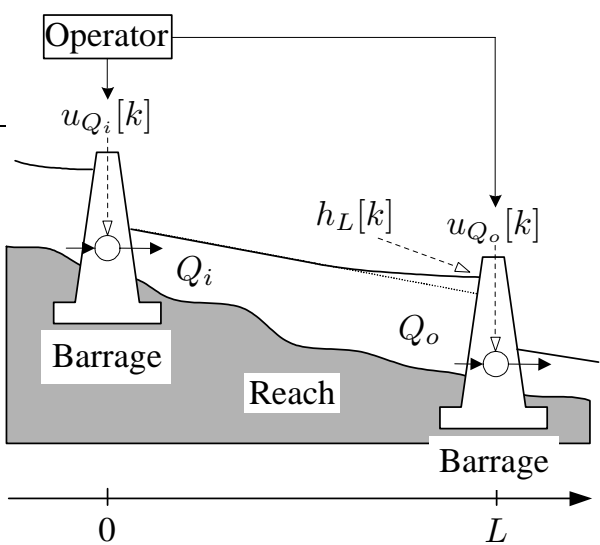

Fig. 1. A managed river reach

Table 1. Main process variables.

\begin{tabular}{cl} 
Reference & Description \\
\hline$u_{Q_{i}}, u_{Q_{o}}$ & Inflow and outflow rate control \\
$Q_{i}, Q_{o}$ & Real inflow and outflow rate \\
$h_{L}$ & Downstream water level \\
$h_{L}^{*}$ & Water level reference \\
$L$ & Length of the reach \\
\hline
\end{tabular}

downstream barrages. The downstream level $h_{L}[k]$ is the controlled variable. The control variable is the outflow rate reference $u_{Q_{o}}[k]$. The inflow rate reference $u_{Q_{i}}[k]$ is considered as a known disturbance. Note that the inflow and outflow rates are not measured. Table 1 gives the main variables of the process.

The problem is to estimate the time-delay between the downstream water level and the inflow rate. This delay depends on the distance between two barrages (typically, several kilometers) and the flow speed. The measurements have been made in passive experimental conditions, i.e. in a normal functioning of the process. In this mode, the difficulty is that the outflow rate reference $u_{Q_{o}}[k]$ is given by a human operator (manual control mode) who adjusts the outflow rate reference in order to control the water level (output) and rejects the disturbances due the inflow rate variations. Of course, this is a feedforward compensation because the operator is able to anticipate level variations. The process has already been modelled in (Thomassin et al., 2003; Thomassin, 2005). The block diagram of the managed river reach is summarized in figure 2 . The two control variables, $u_{Q_{i}}$ and $u_{Q_{o}}$, are correlated, and $u_{Q_{o}}$ is correlated with the noise output.

If we consider the closed-loop structure, we can show that the time-delay of the rational transfer between $u_{Q_{i}}[k]$ and $h_{L}[k]$ is not equal to $d$. So the direct application of Zeng and Feng method is not possible. Consequently, we propose an adaptation of this method in order to estimate the time-delay in the next section.

\section{TIME DELAY ESTIMATION OF A FEEDBACK/FEEDFORWARD STRUCTURE}

The model output is governed by the following relation: 


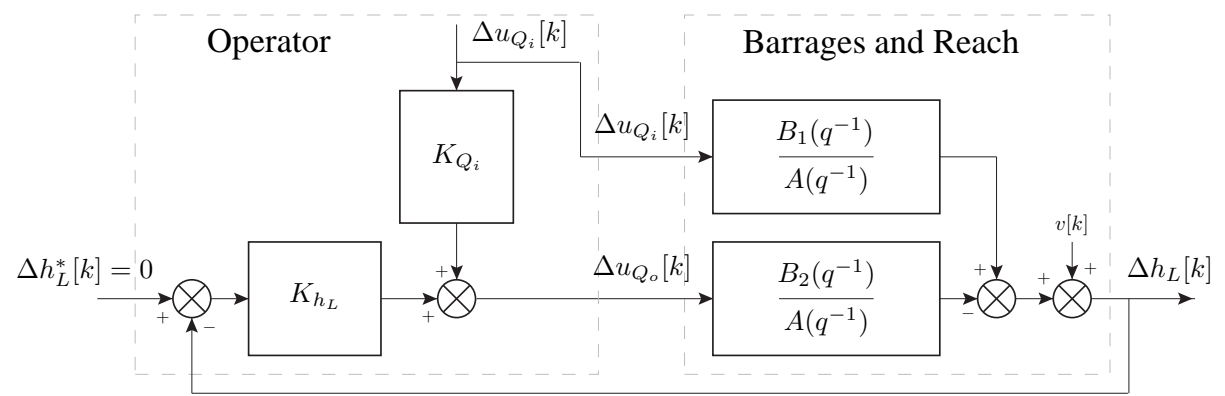

with:

$$
\begin{aligned}
& B_{1}\left(q^{-1}\right)=\frac{K_{i} T_{s}}{A} \\
& B_{2}\left(q^{-1}\right)=\frac{K_{o} T_{s}}{A} \\
& A\left(q^{-1}\right)=1-q^{-1}
\end{aligned}
$$

Fig. 2. Block diagram of a managed river reach.

$$
\begin{aligned}
A\left(q^{-1}\right) y[k]=B_{1}\left(q^{-1}\right) q^{-d} u_{1}[k] & \\
& +B_{2}\left(q^{-1}\right) u_{2}[k]+\omega[k],
\end{aligned}
$$

where

$$
\begin{aligned}
A\left(q^{-1}\right) & =1-a_{1} q^{-1}-\ldots-a_{n_{a}} q^{-n_{a}}, \\
B_{1}\left(q^{-1}\right) & =b_{10}+b_{11} q^{-1}+\ldots+b_{1 n_{b}} q^{-n_{b 1}}, b_{10} \neq 0, \\
B_{2}\left(q^{-1}\right) & =b_{20}+b_{21} q^{-1}+\ldots+b_{2 n_{b}} q^{-n_{b 2}} .
\end{aligned}
$$

The sequences $\left\{u_{1}[k]\right\}_{k=0}^{N-1},\left\{u_{2}[k]\right\}_{k=0}^{N-1}$ and $\{\omega[k]\}_{k=0}^{N-1}$ are stationary and ergodic. The orders $n_{a}, n_{b 1}$ and $n_{b 2}$ are known. Moreover, the following assumptions are assumed to be checked:

- $\mathbf{A}_{\mathbf{1}}:\{\omega[k]\}_{k=0}^{N-1}$ is a white noise of variance $\sigma_{\omega}^{2}$; - $\mathbf{A}_{2}:\left\{u_{1}[k]\right\}_{k=0}^{N-1}$ and $\{\omega[k]\}_{k=0}^{N-1}$ are uncorrelated;

- $\mathbf{A}_{3}:\left\{u_{1}[k]\right\}_{k=0}^{N-1}$ is a MA process of order $n_{f}$ :

$$
u_{1}[k]=F\left(q^{-1}\right) r_{1}[k],
$$

with $F\left(q^{-1}\right)=f_{0}+f_{1} q^{-1}+\ldots+f_{n_{f}} q^{-n_{f}}$ and where $\left\{r_{1}[k]\right\}_{k=0}^{N-1}$ is a uncorrelated random sequence with zero mean and variance $\sigma_{r_{1}}^{2}$;

- $\mathbf{A}_{4}$ : the cross-correlation between $\{\omega[k]\}_{k=0}^{N-1}$ and $\left\{u_{2}[k]\right\}_{k=0}^{N-1}$ is finite:

$$
\left\{\begin{array}{l}
c_{\omega u_{2}}[l] \neq 0, \quad-m \leqslant l \leqslant 0, \\
c_{\omega u_{2}}[l]=0 \quad l<-m \text { or } l>0 ;
\end{array}\right.
$$

- $\mathbf{A}_{\mathbf{5}}$ : the cross-correlation between $\left\{u_{1}[k]\right\}_{k=0}^{N-1}$ et $\left\{u_{2}[k]\right\}_{k=0}^{N-1}$ is finite:

$$
\begin{cases}c_{u_{1} u_{2}}[l] \neq 0, & -p_{1} \leqslant l \leqslant p_{2}, \\ c_{u_{1} u_{2}}[l]=0 & l<-p_{1} \text { or } l>p_{2} .\end{cases}
$$

Then it is shown (see Appendix A) that the generalized Yule-Walker equations are written:

$$
c_{y y}[l]=\sum_{i=1}^{n_{a}} a_{i} c_{y y}[l-i]+\sum_{i=0}^{n_{b 2}} b_{2 i} c_{u_{2} y}[l-i],
$$

for $l>\max \left\{n_{f}-d, p_{2}\right\}+d+n_{b 1}$. They allow to estimate the coefficients $a_{i}$ and $b_{2 i}$.

These estimates permit also to estimate the following statistic:

$$
\begin{aligned}
\hat{\rho}_{y u_{1}}[l] \triangleq c_{y u_{1}}[l]-\sum_{i=1}^{n_{a}} \hat{a}_{i} c_{y u_{1}}[l-i] & { }^{n_{b 2}} \hat{b}_{2 i} c_{u_{2} u_{1}}[l-i], \\
& -\sum_{i=0}
\end{aligned}
$$

which is theoretically equal to:

$$
\begin{aligned}
\rho_{y u_{1}}[l] & =\sum_{i=0}^{n_{b 1}} b_{1 i} c_{u_{1} u_{1}}[l-d-i]+c_{\omega u_{1}}[l], \\
& =\sum_{i=0}^{n_{b 1}} b_{1 i} c_{u_{1} u_{1}}[l-d-i],
\end{aligned}
$$

because $\left\{u_{1}[k]\right\}_{k=0}^{N-1}$ and $\{\omega[k]\}_{k=0}^{N-1}$ are uncorrelated. So this statistic checks the following relations:

$$
\left\{\begin{array}{l}
\rho_{y u_{1}}[l] \neq 0, \quad d-n_{f} \leqslant l \leqslant d+n_{f}+n_{b 1}, \\
\rho_{y u_{1}}[l]=0, \quad l<d-n_{f} \text { or } l>d+n_{f}+n_{b 1} .
\end{array}\right.
$$

Finally, the time-delay $d$ can be estimate by detecting the lag $d-n_{f}$ from which $\rho_{y u_{1}}[l]$ becomes non-null. If the order $n_{f}$ is not known, it can be estimate from the autocorrelation function of $\left\{u_{1}[k]\right\}_{k=0}^{N-1}$.

\section{SIMULATION EXAMPLE}

The aim of this section is to illustrate the previous theory with a numerical example ${ }^{2}$.

Let us consider the following model:

$$
\begin{array}{rl}
y[k]-1,5 & y[k-1]+0,7 y[k-2] \\
= & u_{1}[k-14]+0,5 u_{1}[k-15] \\
& +u_{2}[k]-0,2 u_{2}[k-1]+\omega[k] .
\end{array}
$$

The input $u_{1}$ is described as:

$$
\begin{array}{r}
u_{1}[k]=1,2 r[k]+4,3 r[k-1]+0,5 r[k-2] \\
+0,2 r[k-3]+1,5 r[k-4]+0,3 r[k-5] \\
+0,8 r[k-6]+1,1 r[k-7]+0,4 r[k-8] \\
+2,3 r[k-9],
\end{array}
$$

where $r[k]$ is a pseudorandom binary signal of magnitude 1 . The autocorrelation order, $n_{f}=9$, is assumed to be known. The disturbance $\omega[k]$ is a zero-mean white noise with variance 1 . The time-delay is $d=14$.

2 This example is is close to one of those in (Zheng and Feng, 1990). 
Its range is limited by $d_{\max }=20$. The sample size is $N=8000$. At last, the second input is obtained by:

$$
\begin{aligned}
u_{2}[k]= & u_{1}[k]+\omega[k]+1,3 \omega[k-1]-0,22 \omega[k-2] \\
& -0,832 \omega[k-3]-0,269 \omega[k-4] .
\end{aligned}
$$

We have intentionally chosen a second order model for the river reach, and not an integrator as indicated in figure 2, so as to demonstrate the applicability of our approach in a more general case.

Figure 3 displays the estimate of $\rho_{y u_{1}}$ for one run. It can be seen that $\hat{d}-n_{f}=5$ and $\hat{d}+n_{f}+n_{b 1}=24$, i.e. $\hat{d}=14$. This confirms the result obtained in equation (22). The time-delay and parameter estimates for one run are presented in table 2 . We observe that the estimates of the coefficients $a_{i}$ and $b_{1 i}$ are acceptable. However, we noted that the method does not always lead to good results concerning the estimation of the coefficients $b_{2 i}$. So this point has to be studied further.

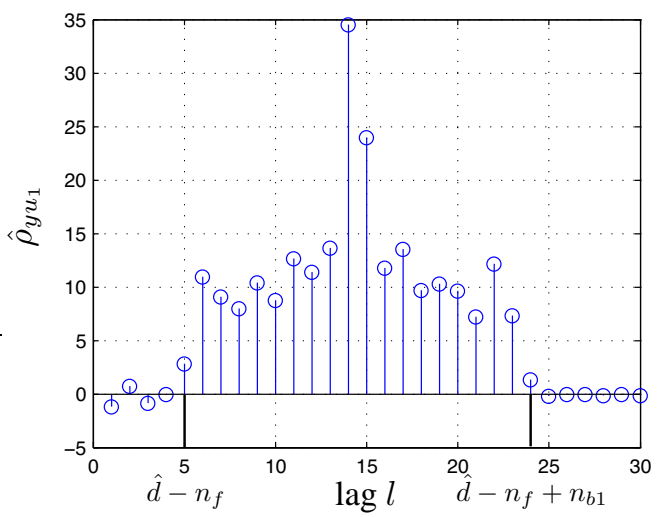

Fig. 3. Estimate of $\rho_{y u_{1}}$ for one run.

Table 2. Parameter estimates for one run.

\begin{tabular}{ccc} 
Parameter & True value & Estimate \\
\hline$d$ & 14 & 14 \\
$a_{1}$ & 1,5 & 1,4415 \\
$a_{2}$ & $-0,7$ & $-0,65826$ \\
$b_{10}$ & 1 & 0,98871 \\
$b_{11}$ & 0,5 & 0,45709 \\
$b_{20}$ & 1 & 1,2537 \\
$b_{21}$ & $-0,2$ & $-0,1362$ \\
\hline
\end{tabular}

The histogram of the time-delay estimates resulting from 100 Monte Carlo runs and obtained with the CUSUM algorithm is shown on figure 4 . It may be seen that $80 \%$ of the estimates correspond to the true time-delay. Concerning the $10 \%$ of detections made at $\hat{d}=19$, they can be reduced by a better tuning of the CUSUM design parameters.

\section{CONCLUSION}

In this paper, a time-delay estimation method in the specific case of structures with combined feedback/feedforward control is presented. It is based on a correlation method developed by Zheng and Feng (1990) in the case of closed-loop structure. Its advantages can be summarized as follows:

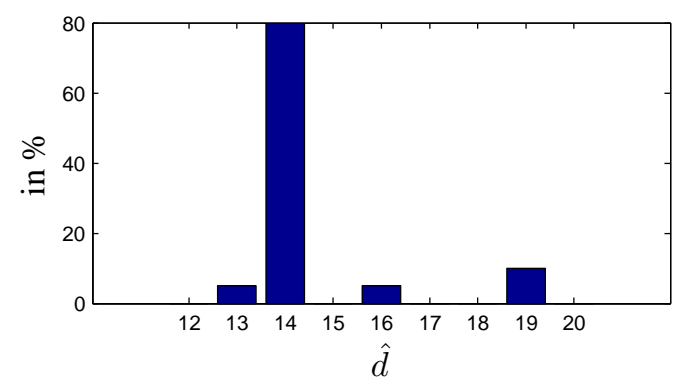

Fig. 4. Estimates of $d$ for 100 Monte Carlo runs.

- no knowledge about the controllers is needed;

- other parameters of the model may be jointly estimated with the time-delay.

This development has been motivated by an application: the estimation of a time-delay of a managed river reach in passive experimental conditions. The theoretical development has been confirmed with a simulation example. As a future work, it would be interesting to study the robustness of the proposed approach in the model coefficients identification. Finally, the development of a recursive version of the algorithm will be considered.

\section{Appendix A. PROOF OF EQUATIONS (18)}

Multiplying equation (14) by $y[k-l]$ and taking the mathematical expectation of the two equality terms leads to:

$$
\begin{array}{r}
c_{y y}[l]=\sum_{i=1}^{n_{a}} a_{i} c_{y y}[l-i]+\sum_{i=0}^{n_{b 1}} b_{1 i} c_{u_{1} y}[l-d-i] \\
+\sum_{i=0}^{n_{b 2}} b_{2 i} c_{u_{1} y}[l-i]+c_{\omega y}[l] .
\end{array}
$$

The aim is to show that:

$$
\sum_{i=0}^{n_{b 1}} b_{1 i} c_{u_{1} y}[l-d-i]+c_{\omega y}[l]=0
$$

for $l>\max \left\{n_{f}-d, p_{2}\right\}+d+n_{b 1}$.

From equation (14), the output $y[k]$ :

$$
\begin{aligned}
y[k] & =\frac{B_{1}\left(q^{-1}\right)}{A\left(q^{-1}\right)} q^{-d} u_{1}[k]+\frac{B_{2}\left(q^{-1}\right)}{A\left(q^{-1}\right)} u_{2}[k] \\
& +\frac{1}{A\left(q^{-1}\right)} \omega[k],
\end{aligned}
$$

can be rewritten in the infinite series form (sum of discrete convolutions):

$$
\begin{aligned}
y[k] & =\sum_{i=0}^{\infty} g_{1}[i] u_{1}[k-d-i]+\sum_{i=0}^{\infty} g_{2}[i] u_{2}[k-i] \\
& +\sum_{i=0}^{\infty} h[i] \omega[k-i] .
\end{aligned}
$$

$\checkmark$ Consider the term $\sum_{i=0}^{n_{b 1}} b_{1 i} c_{u_{1} y}[l-d-i]$ of equation (A.2). From equation (A.4), we obtain: 


$$
\begin{array}{r}
c_{u_{1} y}[l]=\sum_{i=0}^{\infty} g_{1}[i] c_{u_{1} u_{1}}[l+d+i]+\sum_{i=0}^{\infty} g_{2}[i] c_{u_{1} u_{2}}[l+i] \\
+\sum_{i=0}^{\infty} h[i] c_{u_{1} \omega}[l+i]
\end{array}
$$

As $\left\{u_{1}[k]\right\}_{k=0}^{N-1}$ is a MA process of order $n_{f}$, then:

$$
\begin{cases}c_{u_{1} u_{1}} \neq 0, & |l| \leqslant n_{f}, \\ c_{u_{1} u_{1}}=0, & l<-n_{f} \text { or } l>n_{f},\end{cases}
$$

and so we have:

$$
\left\{\begin{array}{l}
\sum_{i=0}^{\infty} g_{1}[i] c_{u_{1} u_{1}}[l+d+i] \neq 0, \quad l \leqslant n_{f}-d \\
\sum_{i=0}^{\infty} g_{1}[i] c_{u_{1} u_{1}}[l+d+i]=0, \quad l>n_{f}-d .
\end{array}\right.
$$

Moreover, since the cross-correlation function between $\left\{u_{1}[k]\right\}_{k=0}^{N-1}$ and $\left\{u_{2}[k]\right\}_{k=0}^{N-1}$ is finite (equation (17)), we get:

$$
\left\{\begin{array}{l}
\sum_{i=0}^{\infty} g_{2}[i] c_{u_{1} u_{2}}[l+i] \neq 0, \quad l \leqslant p_{2}, \\
\sum_{i=0}^{\infty} g_{2}[i] c_{u_{1} u_{2}}[l+i]=0, \quad l>p_{2} .
\end{array}\right.
$$

At least, as we have assumed that $\left\{u_{1}[k]\right\}_{k=0}^{N-1}$ and $\{\omega[k]\}_{k=0}^{N-1}$ are uncorrelated, we obtain:

$$
\sum_{i=0}^{\infty} h[i] c_{u_{1} \omega}[l+i]=0, \quad \forall l .
$$

Using this three results and equation (A.5) gives:

$$
c_{u_{1} y}[l]=0, \quad \text { for } l>\max \left\{n_{f}-d, p_{2}\right\} .
$$

Consequently, we have:

$$
\sum_{i=0}^{n_{b 1}} b_{1 i} c_{u_{1} y}[l-d-i]=0
$$

for $l>n_{b}+d+\max \left\{n_{f}-d, p_{2}\right\}$.

Next consider the term $c_{\omega y}[l]$ in (A.2). Using equation (A.4), we get :

$$
\begin{array}{r}
c_{\omega y}[l]=\sum_{i=0}^{\infty} g_{1}[i] c_{\omega u_{1}}[l+d+i]+\sum_{i=0}^{\infty} g_{2}[i] c_{\omega u_{2}}[l+i] \\
+\sum_{i=0}^{\infty} h[i] c_{\omega \omega}[l+i] .
\end{array}
$$

As $\left\{u_{1}[k]\right\}_{k=0}^{N-1}$ and $\{\omega[k]\}_{k=0}^{N-1}$ are uncorrelated, then:

$$
\sum_{i=0}^{\infty} g_{1}[i] c_{\omega u_{1}}[l+d+i]=0, \quad \forall l .
$$

Moreover, from (16) we obtain:

$$
\left\{\begin{array}{l}
\sum_{i=0}^{\infty} g_{2}[i] c_{\omega u_{2}}[l+i] \neq 0, \quad l \leqslant 0, \\
\sum_{i=0}^{\infty} g_{2}[i] c_{\omega u_{2}}[l+i]=0, \quad l>0 .
\end{array}\right.
$$

At last, as $\{\omega[k]\}_{k=0}^{N-1}$ is a white noise, then:

$$
\left\{\begin{array}{l}
\sum_{i=0}^{\infty} h[i] c_{\omega \omega}[l+i] \neq 0, \quad l \leqslant 0 \\
\sum_{i=0}^{\infty} h[i] c_{\omega \omega}[l+i]=0, \quad l>0 .
\end{array}\right.
$$

Consequently, we get:

$$
c_{\omega y}[l]=0, \quad \text { for } l>0 .
$$

Finally, it follows from (A.10) and (A.15) that the result (A.2) holds, and so the generalized Yule-Walker equations are completely proved.

\section{REFERENCES}

Basseville, M. and I. M. Nikiforov (1993). Detection of abrupt change. Theory and application. Prentice-Hall, Englewood Cliffs, NJ.

Björklund, S. (2003). A survey and comparison of time-delay estimation methods in linear systems. $\mathrm{PhD}$ thesis. Division of Automatic Control, Department of Electrical Engineering, Linköping, Sweden.

Carter, G. C. (1987). Coherence and time delay estimation. Proceedings of the IEEE 75, 236-255.

De Souza, C. E., G. C. Goodwin, D. Q. Mayne and M. Palaniswami (1988). An adaptative control algorithm for linear systems having unknown time delay. Automatica 24(3), 327-341.

Faure, F. and F. J. Evans (1969). Identification of process delay time. IEEE Transactions on Automatic Control AC-14, 421-422.

Ferreira, A. R. and J. M. Fernandes (1997). A survey on time delay system estimation. In: Proceedings of the European Control Conference. Bruxelles, Belgium.

Richard, J.-P. (2003). Time-delay systems: an overview of some recent advances and open problems. Automatica 39, 1667-1694.

Söderström, T. (1999). Feedforward, correlated disturbances and identification. Automatica 35, 15651571.

Thomassin, M. (2005). Estimation de retard dans des conditions expérimentales passives. Application à l'identification d'un bief de rivière. $\mathrm{PhD}$ thesis. Université Henri Poincaré, Nancy 1. France.

Thomassin, M., T. Bastogne, A. Richard and A. Libaux (2003). Time-delay estimation of a managed river reach from supervisory data. In: 13th IFAC Symposium on System Identification. Rotterdam, Netherlands.

Thomassin, M., T. Bastogne, A. Richard and A. Libaux (2004). Estimation des temps de propagation des débits dans les vallées hydrauliques. La Houille Blanche, Revue Internationale de l'Eau 2, 36-42.

Zheng, W.-X. and C.-B. Feng (1990). Identification of stochastic time lag systems in the presence of colored noise. Automatica 26(4), 769-779. 